\title{
Übersicht der palaearktischen Arten der Coleopteren-Gattung Nargus Thoms. aus der Familie der Silphiden.
}

\author{
Von Edm. Reitter in Paskan (Mähren).
}

I" Die Hinterwinkel des Halsschildes sind rechteckig, die Basis ist daneben deutlich ausgebuchtet.

1" Halsschild und Flügeldecken nahezu von gleicher und zwar äußerst feiner und gedrängter Punktur, die Flügeldecken sind nur etwas deutlicher matt. Die ganze Oberseite äußerst dicht und fein staubartig behaart.

2" Körper breit, eiförmig, der Thorax fast breiter als die Flügeldecken, letztere von der Basis zur Spitze stark verengt, mit staubartiger, gelber Behaarung auf der Oberseite. Rostgelb oder gelbbraun, der Kopf meist dunkler braun, seltener sind auch die Flügeldecken schwärzlich. Ein Stück aus Amasia, das ich hierher stellen möchte, ist schwarz, Halsschild und Flügeldecken roströtlich, der Thorax mit schwärzlicher Diskoidalmakel, Fühler und Beine gelbbraun = v. nigriventris nov. - Long. $2 \cdot 7-3 \mathrm{~mm}$.

- Nord- und Mittel-Europa; Spanien.

velox Spence.

?' Körper länglich oval, der Thorax ist höchstens so breit als die Flügeldecken, kürzer, die letzteren länglich, vor der Mitte am breitesten, hell gelbbraun, der Kopf dunkel, der Thorax gewöhnlich in der Mitte mit dunklerem Längswisch.

3" Trochanteren der Hinterbeine des $\sigma^{x}$ spitzig verlängert und die Spitze stark hakenförmig nach innen gebogen; vorletzter Sternit in der Mitte des Spitzenrandes nur schwach ausgebuchtet; Vordertarsen des $\sigma^{x}$ stark erweitert, breiter als die Schienenspitze. Long. $25-3 \mathrm{~mm}$. - Palästina, Transkaukasien, Buchara, Baldschuan, Samarkand.

conjungens Sauley.

3' Trochanteren der Hinterbeine beim $o^{x}$, sowie beim $q$ einfach; vorletztes Sternit in der Mitte des Apicalrandes tiefer rundlich ausgeschnitten, Vordertarsen stark verbreitert. Färbung veränderlich; meist ist diese wie oben angegeben, die Flügeldecken jedoch dunkler braun, manchmal an der Basis heller. - Lon g. $2.8 \mathrm{~mm}$. Palästina, Syrien, Algier. - N. algiricus Portevin, A. 1903, 157.

notaticollis Baudi.

Wiener Entomologische Zeitung, XXV. Jahrg., Heft II, III und IV (15. März 1906). 
1' Halsschild doppelt feiner punktiert als die stärker skulptierten Flügeldecken. Oberseite meist glänzender.

4" Kurz und breit eiförmig, einfarbig braungelb, Halsschild groß und ziemlich lang und fast breiter als die Flügeldecken, die Basis fast gerade; Flügeldecken von der Basis an zur Spitze stark gerundet verengt. Trochanteren der Hinterbeine beim $\sigma^{x}$ wie beim $q$ einfach; Vordertarsen des $\sigma^{7}$ stark verbreitert. - Long. $2.6 \mathrm{~mm}$. -West-Kaukasus: Cirkassien. - W. 1888, 152. ovatus Reitt.

4' Körper stark länglich-oval, Halsschild kürzer, nicht breiter als die Flügeldecken, diese oval.

5" Flügeldecken äußerst fein und dicht, Halsschild kaum erkennbar punktuliert. Dunkel pechbraun, Halsschild heller rostrot, Fühler, Palpen und Beine bräunlichgelb. Trochanteren der Hinterbeine beim $\sigma^{T}$ in einen fast die halbe Schenkellänge erreichenden, doppelt gebogenen Dorn ausgezogen. - Long. $2 \cdot 7 \mathrm{~mm}$.

Kaukasus (Surampass im Meskischen Gebirge).

cribellarius Reitt.

5' Flügeldecken etwas nadelrissig, ziemlich stark, Halsschild fein, auch nadelrissig punktuliert. Einfarbig rostbraun, glänzend, nur die Fühler und Beine heller braungelb. Trochanteren des $\sigma^{x}$ einfach. - Long. 2.5-3 mm. - Mittel-Europa, Schweden. - N. scitulus Thoms.

badius Strm.

I' Hinterwinkel des Halsschildes stumpf oder abgerundet; Basis daneben nicht ausgebuchtet.

a" Halsschild wenig glänzend, dicht und gleichmäßig punktiert, am Grunde zwischen den Punkten chagriniert.

1" Hinterwinkel des Halsschildes äußerst stumpf oder abgerundet. Nicht nur die Mittelschienen leicht gebogen, sondern auch, wenigstens beim $\sigma^{x}$, die Hinterschienen schwach gebogen und ihr Innenrand von der Mitte zur Spitze sehr schwach und breit ausgebuchtet; das Klauenglied des $\sigma^{x}$ an der Basis nach außen zahnförmig erweitert; Vordertarsen beim $\sigma^{x}$ etwas breiter als die Schienenspitze.

2" Schwarzbraun, Fühler, Palpen und Beine braungelb, die Fühlerkeule angedunkelt. Basis des Halsschildes sehr fein und deutlich gerandet, Oberseite gedrängt, fast gleichmäßig, etwas raspelartig punktuliert. - Long. $2 \cdot 6 \mathrm{~mm}$. - Syrien.

Mohammedis Sauley. 
2' Einfarbig hell gelbbraun oder nur der Kopf angedunkelt; Basis des Halsschildes nur in der Mitte und zwar undeutlich gerandet.

3" Gelbbraun, der Kopf dunkler braun. Halsschild gedrängt und sehr fein, die Flügeldecken etwas kräftiger und schwach raspelartig punktuliert. - Long. $3 \mathrm{~mm}$. - Maroceo: Casablanca.

motundangulus Reitt.

3 'Einfarbig braungelb, Oberseite gleichmäßig und außerordentlich fein und gedrängt punktuliert, Seitenrand der Flügeldecken kaum erkennbar abgesetzt. Länglich-oval, fast matt. Fühler kurz, dünn, Glied 4-7 quadratisch, 8-10 quer. Halsschild so breit als die Fłügeldecken zusammen, hinter der Mitte am breitesten, Hinterwinkel angedeutet, nahezu abgerundet, Fiügeldecken lang eiförmig, Nahtstreif wenig stark eingedrückt. Mittelschienen leicht gebogen, die Hinterschienen beim $q$ ger̃ade. ${ }^{1}$ ) - Long. 3 mm. Russisch-Armenien: Araxestal bei Ordubat. Ein $q$ in meiner Kollektion.

densissimus n. sp.

1' Halsschild mit deutlichen stumpfen Hinterwinkeln. Mittelschienen schwach gebogen, Hinterschienen in beiden Geschlechtern einfach und gerade, selten undeutlich gebogen; ebenso ist das Klauenglied des $o^{\top}$ einfach. Vordertarsen beim $o^{\top}$ so breit als die Spitze der Vorderschienen.

4" Hinterschienen beim $\sigma^{x}$ schwach, aber deutlich, beim $q$ undeutlich, aber erkennbar gebogen. Schwarzbraun, Kopf und Halsschild rostrot, die Mitte des letzteren oft getrübt, Fühler und Beine braungelb, die ersteren vor der Spitze angedunkelt; im übrigen ist die Färbung veränderlich und es kommen auch einfarbig braungelbe Individuen vor, nur die Fühler vor der Spitze sind fast immer getrübt. Habituell und in der Punktur der Oberseite den nachfolgenden Arten sehr ähnlich. Die größte Breite des Halsschildes liegt weit hinter der Mitte des stark gerundeten Seitenrandes. - Long. $2 \cdot 6 \mathrm{~mm}$. Corfu; Cephalonia; unter Laub. -- W. 1888, 133.

phaeacus Reitt.

4' Hinterschienen in beiden Geschlechtern gerade. Halsschild fein, Flügeldecken stärker und deutlicher, etwas nadelrissig punktuliert.

5" Halsschild wenig umfangreich, nicht breiter als die Flügeldecken, kurz, reichlich doppelt so breit als lang.

1) Es ist zweifelhaft, ob diese Art hieher gehört, weil das of noch nicht bekannt ist. Der Körperform nach dürfte das $\delta$ mit obigen zwei Arten in nahen Beziehungen stehen.

Wiener Entomologische Zeitung, XXV. Jahrg., Heft II, III und IV (15. März 1906). 
6" Größer, rostrot, der Kopf nicht angedunkelt, die Fühler vor der Spitze, manchmal auch die Scheibe des Halsschildes in der Mitte und selten auch auf den Flügeldecken ein Fleck neben der Naht, getrübt. Hinterwinkel des Halsschildes sehr stumpf, fast abgerundet. -- Long. $2 \cdot 2-2.5 \mathrm{~mm}$. - Griechenland; Norea.

Kraatzi Reitt.

6' Kleiner; rostrot bis schwarz, Kopf immer geschwärzt. — Long. $2-2 \cdot 2 \mathrm{~mm}$.

7" Fühler dünn, vor der Spitze angedunkelt, die Keule unwesentlich breiter, Glied 8 kaum transversal. Rotbraun, der Kopf und die Unterseite schwärzlich, Halsschildmitte und Deckenspitze oft verwaschen getrübt. - Samarkand. turkestanicus n. sp.

7' Fühler dünn, vor der Spitze angedunkelt, die Keule deutlich verbreitert, Glied \& transversal. Basis des Halsschildes sehr deutlich gerandet. Schwarz, Halsschild und Flügeldecken schwarzbraun, Beine braungelb. Oft sind die Seiten des Halsschildes breit roströtlich gesäumt. - Cephalonia. Bei Megalo Vuno von Otto Leonhard zahlreich gesiebt.

Leonhardi n. sp.

5 ' Halsschild breit und groß, merklich breiter als die Flügeldecken, seitlich stark gerundet, nicht ganz doppelt so breit als lang, Hinterwinkel fast rechteckig zulaufend, die Spitze stets abgestumpft. Rostgelb, einfarbig oder mit gebräuntem Kopfe, seltener auch die Scheibe des Halsschildes in der Mitte getrübt, Fühler und Beine hell braungelb. Long. 2.2 $-2.5 \mathrm{~mm}$. - Nord- und Mittel-Europa. - N. praecox Erichs.

Wilkini Spence.

a' Halsschild glänzend, in der Mitte feiner oder spärlicher punktuliert; am Grunde zwischen der Punktur glatt. Halsschild riel feiner punktiert als die Flügeldecken.

1" Hinterwinkel des Halsschildes sehr stumpf, fast abgerundet. Vordertarsen des or so breit als die Schienenspitze.

2" Halsschild nicht doppelt so breit als lang. Langgestreckt, oval, pechbraun, die Beine und Basalhälfte der Fühler gelb. - Long. $3 \mathrm{~mm}$. - Lenkoran.

Ienkoranus Reitt.

2' Halsschild reichlich doppelt so breit als lang.

3" Halsschild fein und deutlich, Flügeldecken doppelt oder dreifach stärker punktiert. Länglich-oval, flach gewölbt, pechbraun, glänzend, Fühler und Beine bräunlichgelb. - Long. 2.5-3 mm. - Dalmatien, Herzegowina, Montenegro, Kleinasien (Bulghar Maaden, Angora).

Nikitanus Reitt. 
3' Kopf und Halsschild fast glatt, Flügeldecken kräftig und dicht raspelartig punktiert. Länglich-oval, glänzend, rotbraun, Fühler, Palpen und Beine hell braungelb. - Long. $24 \mathrm{~mm}$. - Kaukasus. Lebt bei einer Lasius-Art.

Lederi Weise.

1' Hinterwinkel des Halsschildes nahezu rechteckig zulaufend, die Spitze selbst abgestumpft, oder diese sind schwach stumpfeckig, scharf gewinkelt.

4" Vordertarsen des $\sigma^{x}$ erweitert und so breit als die Schienenspitze. Arten von $2 \cdot 2-2 \cdot 5 \mathrm{~mm}$ Länge.

5" Vorderwinkel des Halsschildes sind stumpf, niedergebogen, aber erkennbar. Fühler sehlank und von normaler Länge.

6" Halsschild äußerst fein und weitläufig punktiert, fast glatt. Braunrot, der Kopf dunkler braun, Fühler und Beine hell bräunlichgelb. - Long. $2 \cdot 3$ mm. - Kaukasus. fungicola Kolenati.

6' Halsschild sehr fein und dicht, deutlich punktiert. Rötlich pechbraun, der Kopf und meist auch der Halsschild längs der Mitte dunkler, Fühler und Beine hell bräunlichgelb. _ Long. $2 \cdot 2 \mathrm{~mm}$. Mittel-Europa.

brunneus Strm.

5' Vorderrand des Halsschildes samt den Vorderwinkeln in einer Flucht abgerundet, Halsschild deshalb fast halbkreisförmig, dicht und nur etwas feiner als die Flügeldecken punktiert, die Vorderwinkel nicht angedeutet; Fühler sehr kurz, Glied 4, 5, 7 beim $q$ quadratisch, 6, 9, 10 schwach, 8 stark quer. Sehr kurz oval, gewölbt, pechbraun, der Kopf dunkler braun, Fühler und Beine hell bräunlichgelb. - Long. $2.4 \mathrm{~mm}$. - Kaukasus (Elisabetpol, Helenendorf).

armeniacus Reitt.

4' Vordertarsen des $\sigma^{T}$ schwach erweitert, viel schmäler als die Schienenspitze. Kleine Arten von 1.5 -2 mm Länge.

Oral, gelbrot, glänzend, fein, anliegend behaart. Seltener ist die Beharrung länger und schräg abstehend = var. islamita Reitt. aus Bosnien. - In ganz Europa. anisotomoides spence. 


\section{$2 \mathrm{BHL}$ Biodiversity Heritage Library}

Reitter, Edmund. 1906. "Übersicht der palaearktischen Arten der Coleopteren-Gattung Nargus THOMS. aus der Familie der Silphiden." Wiener entomologische Zeitung 25, 141-145. https://doi.org/10.5962/bhl.part.5384.

View This Item Online: https://www.biodiversitylibrary.org/item/43835

DOI: https://doi.org/10.5962/bhl.part.5384

Permalink: https://www.biodiversitylibrary.org/partpdf/5384

\section{Holding Institution}

Smithsonian Libraries

\section{Sponsored by}

Smithsonian

\section{Copyright \& Reuse}

Copyright Status: NOT_IN_COPYRIGHT

This document was created from content at the Biodiversity Heritage Library, the world's largest open access digital library for biodiversity literature and archives. Visit BHL at https://www.biodiversitylibrary.org. 